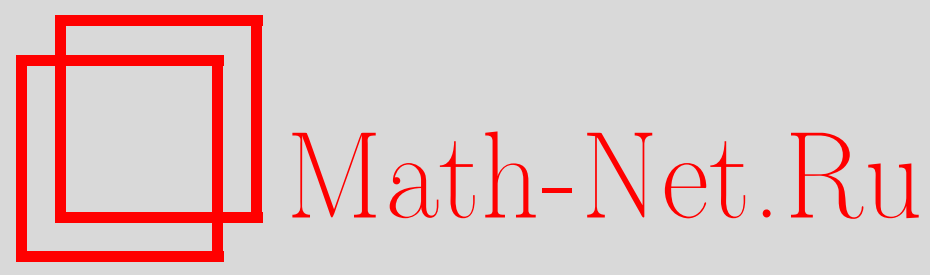

В. Н. Загурский, Н. Т. Воробьев, Классы Фиттинга с заданными свойствами подгрупп Холла, Матем. заметки, 2005, том 78, выпуск 2, 234-240

DOI: https://doi.org/10.4213/mzm2588

Использование Общероссийского математического портала Math-Net.Ru подразумевает, что вы прочитали и согласны с пользовательским соглашением http://www.mathnet.ru/rus/agreement

Параметры загрузки:

IP : 3.85 .7 .115

26 апреля 2023 г., 13:05:52

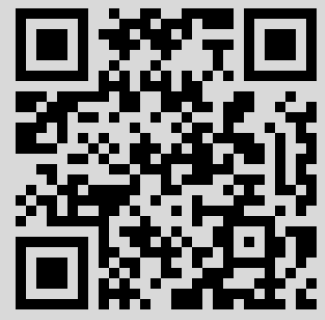




\title{
КЛАССЫ ФИТТИНГА \\ С ЗАДАННЫМИ СВОЙСТВАМИ ПОДГРУПП ХОЛЛА
}

\author{
В. Н. Загурский, Н. Т. Воробьев
}

\begin{abstract}
В теории формаций конечных разрешимых групп известен результат Блессеноля о том, что для любой локальной формации $\mathfrak{F}$ класс всех тех групп, для которых холловская $\pi$-подгруппа принадлежит $\mathfrak{F},-$ также локальная формация. В настоящей работе получен результат, в точности дуальный указанному в теории классов Фиттинга. Доказано, что если класс Фиттинга $\mathfrak{F}$ локален, то и класс всех тех групп, холловские $\pi$-подгруппы которых из $\mathfrak{F}$, также локален.
\end{abstract}

Библиограффия: 8 названий.

Введение. Ряд исследований канонических подгрупп конечных разрешимых групп связан с изучением классов конечных груп, определяемых заданными свойствами подгрупп Холла. В теории формаций хорошо известна своими приложениями для изучения свойств подгруп Холла конструкция класса $B_{\pi}(\mathfrak{X})$ всех тех групп, холловская $\pi$-подгруппа которых принадлежит локальной формации $\mathfrak{X}$. Это обусловлено, прежде всего, результатом Блессеноля [1] о том, что класс $B_{\pi}(\mathfrak{X})$ является локальной формацией для любой локальной формации $\mathfrak{X}$. В теории классов Фиттинга Хауком [2] введена аналогичная конструкция класса $K_{\pi}(\mathfrak{F})$ всех тех групп, холловская $\pi$-подгрупа которых принадлежит классу Фиттинга $\mathfrak{F}$. Известно (см., например, [3, глава 9, п. 1.24]), что такой класс является классом Фиттинга. В дальнейшем Бризоном [4] в терминах класса $K_{\pi}(\mathfrak{F})$ были описаны $\mathfrak{F}$-радикалы холловских $\pi$-подгрупш. Однако вопрос о локальности класса Фиттинга $K_{\pi}(\mathfrak{F})$ оставался открытым. В настоящей работе мы дуализируем указанный результат Блессеноля: доказано, что для любого разрешимого локального класса Фиттинга $\mathfrak{F}$ класс $K_{\pi}(\mathfrak{F})$ является локальным. Более того, установлено, что данный результат справедлив и для частично локальных классов Фиттинга.

Напомним, что если $\pi$ - некоторое множество простых чисел, то через $G_{\pi}$ обозначают холловскую $\pi$-подгрупу групш $G$, т.е. подгруппу, порядок которой есть $\pi$-число, а индекс $-\pi^{\prime}$-число.

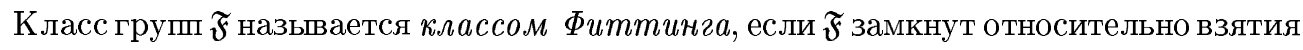
нормальных подгрупп и произведений нормальных $\mathfrak{F}$-подгруп. Из определения следует, что для любого непустого класса Фиттинга $\mathfrak{F}$ в любой группе $G$ существует единственная $\mathfrak{F}$-максимальная нормальная подгруппа $G_{\mathfrak{F}}$ групшы $G$. Ееназывают $\mathfrak{F}-$ радикалом $G$. Через $\mathfrak{F} \mathfrak{H}$ обозначают произведение классов Фиттинга $\mathfrak{F}$ и $\mathfrak{H}$, т.е. класс всех тех групा $G$, для которых $G / G_{\mathfrak{F}} \in \mathfrak{H}$. Хорошо известно, что произведение классов Фиттинга является классом Фиттинга и операция умножения классов Фиттинга ассоциативна. 
Мы будем использовать концепцию частичной локализации Шеметкова-Скибы [5], которая состоит в следующем.

Пусть $\varnothing \neq w \subseteq \mathbb{P}$, где $\mathbb{P}$ - множество всех простых чисел и $w^{\prime}=\mathbb{P} \backslash w$. Всякое отображение

$$
f: w \cup\left\{w^{\prime}\right\} \rightarrow\{\text { классы Фиттинга }\}
$$

назьвается $w$-локальной функиией Хартли или $w$-локальной $H$-функиией [5]. Для каждой $w$-локальной $H$-функции $f$ полагаем

$$
\operatorname{Supp}(f)=\left\{a \in w \cup\left\{w^{\prime}\right\} \mid f(a) \neq \varnothing\right\}
$$

- носитель $f$.

Следуя [5], положим

$$
L R_{w}(f)=\left(\bigcap_{p \in \pi_{2}} \mathfrak{S}_{p^{\prime}}\right) \cap\left(\bigcap_{p \in \pi_{1}} f(p) \mathfrak{N}_{p} \mathfrak{S}_{p^{\prime}}\right) \cap f\left(w^{\prime}\right) \mathfrak{S}_{w}
$$

где $\pi_{1}=\operatorname{Supp}(f) \cap w, \pi_{2}=w \backslash \pi_{1}$ и $\mathfrak{S}_{w}-$ класс всех разрешимых $w$-групп.

Класс Фиттинга $\mathfrak{F}$ называют $w$-локальным $[5]$, если $\mathfrak{F}=L R_{w}(f)$ для некоторой $w$-локальной $H$-функции $f$.

Заметим, что в случае $w=\mathbb{P} w$-локальньй класс Фиттинга назьвают локальным. а $w$-локальную функцию Хартли-локальной функиией Хартли или локальной Н-функиией.

В работе рассматриваются только конечные и разрешимые группы.

Другие определения и обозначения при необходимости можно найти в [3], [6].

1. Свойства класса $K_{\pi}(\mathfrak{F})$. Напомним, что если $\mathfrak{F}-$ класс Фиттинга и $\pi$ - некоторое множество простых чисел, то через $K_{\pi}(\mathfrak{F})$ обозначают класс всех тех групп, в которых холловская $\pi$-подгруппа является $\mathfrak{F}$-группой, т.е.

$$
K_{\pi}(\mathfrak{F})=\left(G \in \mathfrak{S}: G_{\pi} \in \mathfrak{F}\right)
$$

Если $\mathfrak{F}=\varnothing$, то положим $K_{\pi}(\mathfrak{F})=\varnothing$. В случае, когда $\pi=\varnothing$ и $\pi=\mathbb{P}$, положим $K_{\varnothing}(\mathfrak{F})=\mathfrak{S}$ и $K_{\mathbb{P}}(\mathfrak{F})=\mathfrak{F}$ соответственно.

Мы будем неоднократно использовать известные свойства класса $K_{\pi}(\mathfrak{F})$, которые представляет следующая

ЛЕмма 1.1 [4]. Пусть $\mathfrak{F} u \mathfrak{X}-$ классы Фиттинга, $\pi$ - множество простых чисел, $G$ - группа и $G_{\pi}-$ ее холловская $\pi$-подгруппа. Тогда

1) $K_{\pi}(\mathfrak{F} \cap \mathfrak{X})=K_{\pi}(\mathfrak{F}) \cap K_{\pi}(\mathfrak{X}) u$, ecлu $\mathfrak{F} \subseteq \mathfrak{X}$, mo $K_{\pi}(\mathfrak{F}) \subseteq K_{\pi}(\mathfrak{X})$;

2) если $\mathfrak{F}$ - непустой класс, то $G_{K_{\pi}(\mathfrak{F})} \cap G_{\pi}=\left(G_{\pi}\right)_{\mathfrak{F}}$;

3) $K_{\pi}(\mathfrak{F} \mathfrak{X})=K_{\pi}(\mathfrak{F}) K_{\pi}(\mathfrak{X})$.

Лемма 1.2. Пусть $\mathfrak{F}-$ класс Фиттинга и $\pi$-множество простых чисел. Тог$\partial a$

1) если $p \in \pi$, mо $K_{\pi}\left(\mathfrak{S}_{p^{\prime}}\right)=\mathfrak{S}_{p^{\prime}}$

2) если $\mathfrak{F} \neq \varnothing u \mathfrak{F N}_{p}=\mathfrak{F}$ для некоторого простого $p$, то $K_{\pi}(\mathfrak{F}) \mathfrak{N}_{p}=K_{\pi}(\mathfrak{F})$. 
ДоказАтеЛЬСтво. Очевидно, что $\mathfrak{S}_{p^{\prime}} \subseteq K_{\pi}\left(\mathfrak{S}_{p^{\prime}}\right)$. Пусть группа $G \in K_{\pi}\left(\mathfrak{S}_{p^{\prime}}\right)$ и $G_{\pi}$ - холловская $\pi$-подгрупа $G$. Тогда $\left|G_{\pi}\right|$ является $p^{\prime}$-числом. Но $p \in \pi$, и поэтому $\pi^{\prime} \subseteq p^{\prime}$. Следовательно, $G \in \mathfrak{S}_{p^{\prime}}$ и $K_{\pi}\left(\mathfrak{S}_{p^{\prime}}\right) \subseteq \mathfrak{S}_{p^{\prime}}$. Утверждение 1$)$ доказано.

Докажем утверждение 2). Пусть $G \in K_{\pi}(\mathfrak{F}) \mathfrak{N}_{p}$. Так как по утверждению 2) леммы $1.1 G_{\pi} \cap G_{K_{\pi}(\mathfrak{F})}=\left(G_{\pi}\right)_{\mathfrak{F}}$, то ввиду изоморфизма

$$
G_{\pi} G_{K_{\pi}(\mathfrak{F})} / G_{K_{\pi}(\mathfrak{F})} \cong G_{\pi} /\left(G_{\pi} \cap G_{K_{\pi}(\mathfrak{F})}\right)=G_{\pi} /\left(G_{\pi}\right)_{\mathfrak{F}}
$$

имеем

$$
G_{\pi} /\left(G_{\pi}\right)_{\mathfrak{F}} \cong G_{\pi} G_{K_{\pi}(\mathfrak{F})} / G_{K_{\pi}(\mathfrak{F})} .
$$

Но холловская $\pi$-подгруша $G_{\pi} G_{K_{\pi}(\mathfrak{F})} / G_{K_{\pi}(\mathfrak{F})}$ групшы $G / G_{K_{\pi}(\mathfrak{F})}$ является $p$-группой. Следовательно, $G_{\pi} /\left(G_{\pi}\right)_{\mathfrak{F}} \in \mathfrak{N}_{p}$ и $G_{\pi} \in \mathfrak{F}$, и справедливо включение

$$
K_{\pi}(\mathfrak{F}) \mathfrak{N}_{p} \subseteq K_{\pi}(\mathfrak{F}) .
$$

Обратное включение очевидно. Лемма доказана.

Лемма 1.3. Пусть $\mathfrak{F}$ - непустой класс Фиттинга $и \pi, \sigma$ - множества простых чисел, причем $\sigma \cap \pi=\varnothing$. Тогда

$$
K_{\sigma}(\mathfrak{F}) \mathfrak{S}_{\pi}=K_{\sigma}(\mathfrak{F}) .
$$

ДоКАЗАТЕЛЬСТво. Очевидно, что $K_{\sigma}(\mathfrak{F}) \subseteq K_{\sigma}(\mathfrak{F}) \mathfrak{S}_{\pi}$.

Пусть $G \in K_{\sigma}(\mathfrak{F}) \mathfrak{S}_{\pi}$. По утверждению 2) леммы $1.1 G_{\sigma} \cap G_{K_{\sigma}(\mathfrak{F})}=\left(G_{\sigma}\right)_{\mathfrak{F}}$. Учитывая изоморфизм

$$
G_{\sigma} G_{K_{\sigma}(\mathfrak{F})} / G_{K_{\sigma}(\mathfrak{F})} \cong G_{\sigma} /\left(G_{\sigma} \cap G_{K_{\sigma}(\mathfrak{F})}\right)=G_{\sigma} /\left(G_{\sigma}\right)_{\mathfrak{F}}
$$

получаем

$$
G_{\sigma} /\left(G_{\sigma}\right)_{\mathfrak{F}} \cong G_{\sigma} G_{K_{\sigma}(\mathfrak{F})} / G_{K_{\sigma}(\mathfrak{F})} .
$$

Но тогда из того, что $G / G_{K_{\sigma}(\mathfrak{F})} \in \mathfrak{S}_{\pi}$, следует $G_{\sigma} /\left(G_{\sigma}\right)_{\mathfrak{F}} \in \mathfrak{S}_{\pi}$ и поэтому $G_{\sigma}=\left(G_{\sigma}\right)_{\mathfrak{F}}$. Следовательно, $G_{\sigma} \in \mathfrak{F}$ и $G \in K_{\sigma}(\mathfrak{F})$. Лемма доказана.

СледСТвИЕ 1.4. Пусть $\mathfrak{F}$ - непустой класс Фиттинга $и \pi, \sigma$ - множества простых чисел, причем $\sigma \cap \pi=\varnothing$. Тогда

$$
K_{\sigma}(\mathfrak{F}) \mathfrak{N}_{\pi}=K_{\sigma}(\mathfrak{F}) .
$$

ДоКАЗАТЕЛЬСТВо следует из того, что по лемме 1.3

$$
K_{\sigma}(\mathfrak{F}) \mathfrak{N}_{\pi} \subseteq K_{\sigma}(\mathfrak{F}) \mathfrak{S}_{\pi}=K_{\sigma}(\mathfrak{F}) .
$$

СлЕДСТвИЕ 1.5. Если $\mathfrak{F}$ - непустой класс Фиттинга и $\pi$-множество простых чисел, mo

$$
K_{\pi}(\mathfrak{F}) \mathfrak{S}_{\pi^{\prime}}=K_{\pi}(\mathfrak{F})
$$


2. Локальность класса $K_{\pi}(\mathfrak{F})$. Основной результат настоящей работы представляет следующая

ТЕОРема 2.1. Если $\mathfrak{F}-$ - -локальный класс Фиттинга и $\pi$-некоторое множество простых чисел, то $K_{\pi \cap w}(\mathfrak{F})$ - w-локальный класс Фиттинга.

ДоказАТЕЛьство. Допустим, что $\mathfrak{F}=\varnothing$. Так как класс Фиттинга $\varnothing$ является $w$-локальным (см., $[5$, пример 10$])$, то класс $K_{\pi \cap w}(\varnothing) w$-локален и в этом случае теорема справедлива.

Предположим, что $\mathfrak{F} \neq \varnothing$. Если $w \cap \pi=\varnothing$, то по определению $K_{\varnothing}(\mathfrak{F})=\mathfrak{S}$. Определим $H$-функцию следующим образом: $f(a)=\mathfrak{S}$ для всех $a$ из $w \cup\left\{w^{\prime}\right\}$. Тогда, очевидно,

$$
L R_{w}(f)=\left(\bigcap_{p \in w} \mathfrak{S N}_{p} \mathfrak{S}_{p^{\prime}}\right) \cap \mathfrak{S S}_{w}=\mathfrak{S}
$$

и класс Фиттинга $K_{\pi \cap w}(\mathfrak{F}) w$-локален.

Предположим, что $w \cap \pi \neq \varnothing$. Так как $\mathfrak{F}-w$-локальный класс Фиттинга, то по теореме 9 из [5] существует $w$-локальная $H$-функция $F$ такая, что $\mathfrak{F}=L R_{w}(F)$, ее значения $F(p)=F(p) \mathfrak{N}_{p} \subseteq \mathfrak{F}$ для всех $p$ из $w$ и $F\left(w^{\prime}\right)=\mathfrak{F}$.

Обозначим

$$
\pi_{1}=\operatorname{Supp}(F) \cap w, \quad \pi_{2}=w \backslash \pi_{1}
$$

Тогда

$$
\mathfrak{F}=L R_{w}(F)=\left(\bigcap_{p \in \pi_{2}} \mathfrak{S}_{p^{\prime}}\right) \cap\left(\bigcap_{p \in \pi_{1}} F(p) \mathfrak{N}_{p} \mathfrak{S}_{p^{\prime}}\right) \cap \mathfrak{F} \mathfrak{S}_{w}
$$

Построим $w$-локальную $H$-функцию следующим образом:

$$
f(p)= \begin{cases}K_{\pi_{1} \cap \pi}(F(p)), & \text { если } p \in \pi_{1} \cap \pi ; \\ K_{\pi \cap w}(\mathfrak{F}), & \text { если } p \in w \backslash \pi ; \\ \varnothing, & \text { если } p \in \pi_{2} \cap \pi\end{cases}
$$

и $f\left(w^{\prime}\right)=K_{\pi \cap w}(\mathfrak{F})$.

Легко видеть, что $\operatorname{Supp}(f)=\left(\pi_{1} \cap \pi\right) \cup(w \backslash \pi) \cup\left\{w^{\prime}\right\}$. Следовательно,

$$
\begin{aligned}
L R_{w}(f)= & \left(\bigcap_{p \in \pi_{2} \cap \pi} \mathfrak{S}_{p^{\prime}}\right) \cap\left(\bigcap_{p \in \pi_{1} \cap \pi} K_{\pi_{1} \cap \pi}(F(p)) \mathfrak{N}_{p} \mathfrak{S}_{p^{\prime}}\right) \\
& \cap\left(\bigcap_{p \in w \backslash \pi} K_{\pi \cap w}(\mathfrak{F}) \mathfrak{N}_{p} \mathfrak{S}_{p^{\prime}}\right) \cap K_{\pi \cap w}(\mathfrak{F}) \mathfrak{S}_{w} .
\end{aligned}
$$

Покажем вначале, что справедливо равенство

$$
\bigcap_{p \in \pi_{1} \cap \pi} K_{\pi_{1} \cap \pi}(F(p)) \mathfrak{N}_{p} \mathfrak{S}_{p^{\prime}}=K_{\pi_{1} \cap \pi}(\mathfrak{F}) .
$$

Так как $\mathfrak{F}-w$-локальньй класс Фиттинга и $F(p)=F(p) \mathfrak{N}_{p}$ для всех $p$ из $w$, то $\mathfrak{F} \subseteq \bigcap_{p \in \pi_{1} \cap \pi} F(p) \mathfrak{S}_{p^{\prime}}$. Тогда по утверждению 1) леммы 1.1

$$
K_{\pi_{1} \cap \pi}(\mathfrak{F}) \subseteq K_{\pi_{1} \cap \pi}\left(\bigcap_{p \in \pi_{1} \cap \pi} F(p) \mathfrak{S}_{p^{\prime}}\right)=\bigcap_{p \in \pi_{1} \cap \pi} K_{\pi_{1} \cap \pi}\left(F(p) \mathfrak{S}_{p^{\prime}}\right)
$$


Следовательно, по утверждению 3) леммы 1.1 и утверждению 1) леммы 1.2 получаем

$$
\bigcap_{p \in \pi_{1} \cap \pi} K_{\pi_{1} \cap \pi}\left(F(p) \mathfrak{S}_{p^{\prime}}\right)=\bigcap_{p \in \pi_{1} \cap \pi} K_{\pi_{1} \cap \pi}(F(p)) \mathfrak{S}_{p^{\prime}}
$$

Кроме того, по утверждению 2) леммы 1.2 для любого $p \in \pi_{1} \cap \pi$ имеет место

$$
K_{\pi_{1} \cap \pi}(F(p)) \subseteq K_{\pi_{1} \cap \pi}(F(p)) \mathfrak{N}_{p}
$$

Значит,

$$
K_{\pi_{1} \cap \pi}(\mathfrak{F}) \subseteq \bigcap_{p \in \pi_{1} \cap \pi} K_{\pi_{1} \cap \pi}(F(p)) \mathfrak{N}_{p} \mathfrak{S}_{p^{\prime}}
$$

Докажем обратное включение. Действительно, из того, что $F(p) \subseteq \mathfrak{F}$ для всех $p$ из $w$, из утверждения 1) леммы 1.1 следует включение

$$
K_{\pi_{1} \cap \pi}(F(p)) \subseteq K_{\pi_{1} \cap \pi}(\mathfrak{F})
$$

Значит,

$$
K_{\pi_{1} \cap \pi}(F(p)) \mathfrak{S}_{p^{\prime}} \subseteq K_{\pi_{1} \cap \pi}(\mathfrak{F}) \mathfrak{S}_{p^{\prime}}
$$

и по утверждению 2) леммы 1.2

$$
K_{\pi_{1} \cap \pi}(F(p))=K_{\pi_{1} \cap \pi}(F(p)) \mathfrak{N}_{p}
$$

для любого $p$ из $\pi_{1} \cap \pi$. Следовательно,

$$
\bigcap_{p \in \pi_{1} \cap \pi} K_{\pi_{1} \cap \pi}(F(p)) \mathfrak{N}_{p} \mathfrak{S}_{p^{\prime}} \subseteq \bigcap_{p \in \pi_{1} \cap \pi} K_{\pi_{1} \cap \pi}(\mathfrak{F}) \mathfrak{S}_{p^{\prime}}=K_{\pi_{1} \cap \pi}(\mathfrak{F}) \mathfrak{S}_{\left(\pi_{1} \cap \pi\right)^{\prime}}
$$

Кроме того, по следствию 1.5

$$
K_{\pi_{1} \cap \pi}(\mathfrak{F}) \mathfrak{S}_{\left(\pi_{1} \cap \pi\right)^{\prime}}=K_{\pi_{1} \cap \pi}(\mathfrak{F}) .
$$

Значит,

$$
\bigcap_{p \in \pi_{1} \cap \pi} K_{\pi_{1} \cap \pi}(F(p)) \mathfrak{N}_{p} \mathfrak{S}_{p^{\prime}} \subseteq K_{\pi_{1} \cap \pi}(\mathfrak{F})
$$

и равенство (1) доказано.

Установим теперь, что

$$
\bigcap_{p \in w \backslash \pi} K_{\pi \cap w}(\mathfrak{F}) \mathfrak{N}_{p} \mathfrak{S}_{p^{\prime}}=K_{\pi \cap w}(\mathfrak{F}) \mathfrak{S}_{(w \backslash \pi)^{\prime}}
$$

По лемме $1.3 K_{\pi \cap w}(\mathfrak{F})=K_{\pi \cap w}(\mathfrak{F}) \mathfrak{S}_{w \backslash \pi}$. Кроме того, $\mathfrak{S}_{w \backslash \pi} \mathfrak{N}_{p}=\mathfrak{S}_{w \backslash \pi}$ для любого $p$ из $w \backslash \pi$. Следовательно,

$$
\bigcap_{p \in w \backslash \pi} K_{\pi \cap w}(\mathfrak{F}) \mathfrak{N}_{p} \mathfrak{S}_{p^{\prime}}=\bigcap_{p \in w \backslash \pi} K_{\pi \cap w}(\mathfrak{F}) \mathfrak{S}_{w \backslash \pi} \mathfrak{N}_{p} \mathfrak{S}_{p^{\prime}}=\bigcap_{p \in w \backslash \pi} K_{\pi \cap w}(\mathfrak{F}) \mathfrak{S}_{w \backslash \pi} \mathfrak{S}_{p^{\prime}}
$$


И

$$
\bigcap_{p \in w \backslash \pi} K_{\pi \cap w}(\mathfrak{F}) \mathfrak{S}_{w \backslash \pi} \mathfrak{S}_{p^{\prime}}=\bigcap_{p \in w \backslash \pi} K_{\pi \cap w}(\mathfrak{F}) \mathfrak{S}_{p^{\prime}}=K_{\pi \cap w}(\mathfrak{F}) \mathfrak{S}_{(w \backslash \pi)^{\prime}}
$$

что доказьвает равенство (2).

Таким образом, мы доказали справедливость равенства

$$
L R_{w}(f)=\mathfrak{S}_{\left(\pi_{2} \cap \pi\right)^{\prime}} \cap K_{\pi_{1} \cap \pi}(\mathfrak{F}) \cap K_{\pi \cap w}(\mathfrak{F}) \mathfrak{S}_{(w \backslash \pi)^{\prime}} \cap K_{\pi \cap w}(\mathfrak{F}) \mathfrak{S}_{w}
$$

Теперь, учитьвая (3), для доказательства теоремы достаточно выяснить справедливость равенства

$$
\mathfrak{S}_{\left(\pi_{2} \cap \pi\right)^{\prime}} \cap K_{\pi_{1} \cap \pi}(\mathfrak{F})=K_{\pi \cap w}(\mathfrak{F})
$$

Пусть $G \in K_{\pi \cap w}(\mathfrak{F})$. Тогда из того, что $\mathfrak{F}=L R_{w}(F)$, следует $G_{\pi \cap w} \in \mathfrak{F} \subseteq \mathfrak{S}_{\pi_{2}^{\prime}}$ и поэтому $\left|G_{\pi \cap w}\right|$ является $\left(\pi_{2}^{\prime} \cap \pi \cap w\right)$-числом. Кроме того, $\left|G: G_{\pi \cap w}\right|$ является $(\pi \cap w)^{\prime}$-числом. Легко видеть, что $(\pi \cap w)^{\prime} \cup\left(\pi_{2}^{\prime} \cap \pi \cap w\right)=\left(\pi_{2} \cap \pi\right)^{\prime}$ и, значит, $G \in \mathfrak{S}_{\left(\pi_{2} \cap \pi\right)^{\prime}}$. Так как $\pi_{1} \subseteq w$, то $(\pi \cap w)^{\prime} \subseteq\left(\pi_{1} \cap \pi\right)^{\prime}$ и $\left|G: G_{\pi \cap w}\right|$ является $\left(\pi_{1} \cap \pi\right)^{\prime}$-числом. Далее из $\pi_{1} \cap \pi \cap w \subseteq \pi_{1} \cap \pi$ следует, что $\left|G_{\pi \cap w}\right|$ есть $\left(\pi_{1} \cap \pi\right)$-число. Тогда $(\pi \cap w)$-холловская подгрупша $G_{\pi \cap w}$ группы $G$ является также $\left(\pi_{1} \cap \pi\right)$-холловской подгруппой групшы $G$. Значит, $G_{\pi_{1} \cap \pi} \in \mathfrak{F}$ и $G \in K_{\pi_{1} \cap \pi}(\mathfrak{F})$. Следовательно,

$$
K_{\pi \cap w}(\mathfrak{F}) \subseteq \mathfrak{S}_{\left(\pi_{2} \cap \pi\right)^{\prime}} \cap K_{\pi_{1} \cap \pi}(\mathfrak{F})
$$

Покажем обратное включение. Пусть теперь $G \in \mathfrak{S}_{\left(\pi_{2} \cap \pi\right)^{\prime}} \cap K_{\pi_{1} \cap \pi}(\mathfrak{F})$. Тогда $G_{\pi_{1} \cap \pi} \in \mathfrak{F}$. Так как $\left(\pi_{2} \cap \pi\right)^{\prime} \backslash\left(\pi_{1} \cap \pi\right)=(\pi \cap w)^{\prime}$, то $\left|G: G_{\pi_{1} \cap \pi}\right|$ является $(\pi \cap w)^{\prime}$-числом. Теперь, ввиду $\pi_{1} \cap \pi \subseteq \pi \cap w$, получаем, что $\left|G_{\pi_{1} \cap \pi}\right|$ является $(\pi \cap w)$-числом. Значит, $\left(\pi_{1} \cap \pi\right)$-холловская подгруппа $G$ является $(\pi \cap w)$-холловской подгруппой $G$ и поэтому $G_{\pi \cap w} \in \mathfrak{F}$. Следовательно, $G \in K_{\pi \cap w}(\mathfrak{F})$. Это означает, что

$$
\mathfrak{S}_{\left(\pi_{2} \cap \pi\right)^{\prime}} \cap K_{\pi_{1} \cap \pi}(\mathfrak{F}) \subseteq K_{\pi \cap w}(\mathfrak{F})
$$

и равенство (4) доказано.

Теорема доказана.

В случае $w=\mathbb{P}$ из теоремы вытекает результат, в точности дуальньй указанному во введении результату Блессеноля [1].

СЛЕДСТВИЕ 2.2. Если $\mathfrak{F}$ - локальный класс Фиттинга и тво простых чисел, то $K_{\pi}(\mathfrak{F})$ - локальный класс Фиттинга.

Из следствия вытекает, что семейство всех тех классов Фиттинга $\mathfrak{F}$, для которых $K_{\pi}(\mathfrak{F})$ является локальным, обширно, так как оно содержит все локальные классы Фиттинга. Однако в общем случае класс Фиттинга $K_{\pi}(\mathfrak{F})$ локален не для всякого класса Фиттинга $\mathfrak{F}$. Это подтверждает следующий 
ПРимеР 2.3. Пусть $\mathfrak{F}$ - произвольньй нетривиальный (отличный от классов (1) и $\mathfrak{S}$ ) нормальный класс Фиттинга. Покажем, что $\mathfrak{F}$ нелокален. Действительно, если $\mathfrak{F}$ локален, то по лемме 5 из [7] $\mathfrak{F}$ является классом Локетта и поэтому ввиду [3, теорема Х.3.7] $\mathfrak{F}^{*}=\mathfrak{F}=\mathfrak{S}$. Полученное противоречие показывает, что любой нетривиальный нормальный класс Фиттинга нелокален.

Пусть теперь $p$ и $q$ - такие простые числа, что $p \mid(q-1), G=D_{q^{n}}^{p}$-монолитическая группа с нормальной абелевой силовской $q$-подгруппой экспоненты $q^{n}$ и циклической холловской $q^{\prime}$-подгруппой порядка $p$. Пусть $\pi=\pi(G)$ и $\mathfrak{S}_{*}-$ минимальньй нормальный класс Фиттинга. Тогда, ввиду результата Бергера (см. свойство 3 из [8]) группа $G \notin \mathfrak{S}_{*}$ и поэтому $G \notin K_{\pi}\left(\mathfrak{S}_{*}\right)$. Следовательно, $K_{\pi}\left(\mathfrak{S}_{*}\right) \neq \mathfrak{S}$ и класс Фиттинга $K_{\pi}\left(\mathfrak{S}_{*}\right)$ нетривиален. Но по теореме Хаука (см. [2, теорема 3.4$\left.]\right) K_{\pi}\left(\mathfrak{S}_{*}\right)$ - нормальный класс Фиттинга и, следовательно, нелокален.

В заключение, заметим, что если $\mathfrak{F}$ - непустой $w$-локальный класс Фиттинга и $\pi$ некоторое множество простых чисел, причем $w \cap \pi=\varnothing$, то класс $K_{\pi}(\mathfrak{F})$ является также $w$-локальным. Действительно, в этом случае определяя значения $w$-локальной $H$-функции равенством $f(a)=K_{\pi}(\mathfrak{F})$ для всех $a$ из $w \cup\left\{w^{\prime}\right\}$, получаем, что

$$
L R_{w}(f)=\left(\bigcap_{p \in w} K_{\pi}(\mathfrak{F}) \mathfrak{N}_{p} \mathfrak{S}_{p^{\prime}}\right) \cap K_{\pi}(\mathfrak{F}) \mathfrak{S}_{w}=K_{\pi}(\mathfrak{F}) \mathfrak{N}_{w} \mathfrak{S}_{w^{\prime}} \cap K_{\pi}(\mathfrak{F}) \mathfrak{S}_{w}
$$

Теперь, учитывая следствие 1.4 , получаем, что $K_{\pi}(\mathfrak{F}) \mathfrak{N}_{w}=K_{\pi}(\mathfrak{F})$ и

$$
L R_{w}(f)=K_{\pi}(\mathfrak{F}) \mathfrak{S}_{w^{\prime}} \cap K_{\pi}(\mathfrak{F}) \mathfrak{S}_{w}=K_{\pi}(\mathfrak{F})\left(\mathfrak{S}_{w^{\prime}} \cap \mathfrak{S}_{w}\right)=K_{\pi}(\mathfrak{F})
$$

\section{СПИСОК ЦИТИРОВАННОЙ ЛИТЕРАТУРЫ}

[1] Blessenohl D. Über Formationen und Halluntergruppen endlicher auflösbarer Gruppen // Math. Z. 1975. V. 142. № 3. P. 299-300.

[2] Hauck P. Eine Bemerkung zur kleinsten normalen Fittingklasse // J. Algebra. 1978. V. 53. P. 395-401.

[3] Doerk K., Hawkes T. Finite Soluble Groups. Berlin-New York: Walter de Gruyter, 1992.

[4] Brison O. J. Hall operators for Fitting classes // Arch. Math. (Basel). 1979. V. 33. P. 1-9.

[5] Скиба А. Н., Шеметков Л. А. Кратно локальные формации и классы Фиттинга конечных групп // Матем. труды. 1999. Т. 2. № 2. С. 114-147.

[6] Шеметков Л. А. Формации конечных групп. М.: Наука, 1978.

[7] Воробьев Н. Т. О радикальных классах конечных групп с условием Локетта // Матем. заметки. 1988. Т. 43. №2. С. 161-168.

[8] Berger T. K. More normal Fitting classes of finite solvable groups // Math. Z. 1976. V. 151. №1. P. 1-3. 